Article

\title{
Operations on Oriented Maps
}

\author{
Tomaž Pisanski ${ }^{1, *}$, Gordon Williams ${ }^{2}$ (D) and Leah Wrenn Berman ${ }^{2}$ \\ 1 Department of Information Sciences and Technologies (FAMNIT), University of Primorska, \\ 6000 Koper, Slovenia \\ 2 Department of Mathematics \& Statistics, University of Alaska Fairbanks, Fairbanks, AK 99775, USA; \\ giwilliams@alaska.edu (G.W.); lwberman@alaska.edu (L.W.B.) \\ * Correspondence: Tomaz.Pisanski@upr.si
}

Received: 31 July 2017; Accepted: 11 November 2017; Published: 14 November 2017

\begin{abstract}
A map on a closed surface is a two-cell embedding of a finite connected graph. Maps on surfaces are conveniently described by certain trivalent graphs, known as flag graphs. Flag graphs themselves may be considered as maps embedded in the same surface as the original graph. The flag graph is the underlying graph of the dual of the barycentric subdivision of the original map. Certain operations on maps can be defined by appropriate operations on flag graphs. Orientable surfaces may be given consistent orientations, and oriented maps can be described by a generating pair consisting of a permutation and an involution on the set of arcs (or darts) defining a partially directed arc graph. In this paper we describe how certain operations on maps can be described directly on oriented maps via arc graphs.
\end{abstract}

Keywords: map; oriented map; truncation; dual; medial; snub; flag graph; arc graph

MSC: 52C20, 05C10, 51M20

\section{Maps and Oriented Maps}

A map $\mathcal{M}$ on a closed surface is a two-cell embedding of a finite connected graph $G=(V, E)$ (see, e.g., [1] or [2]). Equivalently, every map can be viewed as a set of three fixed-point-free involutions $r_{0}, r_{1}$ and $r_{2}$ acting on a set of flags $\Omega$ with the property that $r_{0} r_{2}$ is also a fixed-point-free involution, in which case we denote the map as $\mathcal{M}=\left(\Omega,\left\langle r_{0}, r_{1}, r_{2}\right\rangle\right)$; see, for instance, [3] (p. 415). With this second point of view, each map can be described completely using a three-edge colored cubic graph, called the flag graph, whose vertices are elements of $\Omega$, where $\omega_{1}$ and $\omega_{2}$ are connected by an edge colored $i$ in the flag graph if and only if $r_{i}\left(\omega_{1}\right)=\omega_{2}$. Generally, only connected flag graphs are considered. The graph $G$ is called the skeleton or the underlying graph of a map $\mathcal{M}$.

In some cases, one may relax the conditions and allow fixed points in some of the involutions $r_{0}, r_{1}$ and $r_{2}$. Such a structure describes a map in a surface with a boundary and with its flag graph containing semi-edges. We call such a map and respective flag graph degenerate.

If the surface in which the map resides is orientable, then $\mathcal{M}$ is said to be an orientable map. There is a well-known test involving flag graphs to determine whether a given map is orientable:

Proposition 1. A map is orientable if and only if its flag graph is bipartite.

In a bipartite flag graph, the flags (i.e., vertices) of the map come in two color classes; the vertices in a single color class of flags are called arcs. Restricting attention to one set of arcs corresponds to choosing an orientation of the orientable map, and we call the restricted graph an oriented map; each orientable map gives rise to two oppositely oriented oriented maps. We note that in a map there are four flags per edge of the skeleton, while in an oriented map there are two arcs per edge. That is, 
if $\Omega$ denotes the set of flags of the flag graph, and $\Sigma$ denotes one of the sets of arcs of the oriented graph, then $|\Omega|=4|E|$ and $|\Sigma|=2|E|$, where $E$ is the set of edges of the skeleton of the original map.

Considering only oriented maps, as we do in this paper, allows us to treat each of the two orientations of an orientable map separately. It also allows us to introduce the notion of an oriented symmetry type graph, which captures the symmetry properties that are preserved under orientation-preserving automorphisms of an oriented map. Most researchers consider maps and oriented maps separately. A prominent exception is found in the lecture notes of Roman Nedela (see [4]), where both maps and oriented maps are studied using the same tools. The main difference between our approach and Nedela's is that Nedela puts the emphasis on groups, while we focus mainly on graphs (flag graphs and arc graphs).

More technically, we define an oriented map to be a structure $\mathcal{M}_{0}:=\{\Sigma,\langle r, R\rangle\}$, where $r$ is a fixed-point-free involution and $R$ is any permutation acting on a set of objects $\Sigma$, called arcs. We also view the oriented map $\mathcal{M}_{o}$ as a partially directed graph, called an arc graph, whose vertices are elements of $\Sigma$, having some edges directed and some undirected. The directed edges form a directed 2 -factor, corresponding to the action of the permutation $R$ on elements of $\Sigma$. The undirected edges form an undirected 1-factor, corresponding to the action of the involution $r$ on the elements of $\Sigma$. Again, the only other constraint usually imposed on such a structure is the fact that the arc graph must be connected. If the involution $r$ is not fixed-point-free, then we say that the corresponding arc graph is degenerate.

We note that each oriented map $\mathcal{M}_{0}$ gives rise to its skeleton graph $G$ in the following way: the vertices of $G$ are the orbits of $R$ on $\Sigma$ and the edges of $G$ are the orbits of $r$ on $\Sigma$. A vertex is incident with an edge if and only if the corresponding orbits have a non-empty intersection. Using this description, we can generate an oriented map $\mathcal{M}_{0}$ from any orientable map $\mathcal{M}=\left\{\Omega,\left\langle r_{0}, r_{1}, r_{2}\right\rangle\right\}$ by first observing that, because $\mathcal{M}$ is orientable, the flag graph is bipartite, with bipartite sets $\Omega^{+}$and $\Omega^{-}$. We define $\Sigma=\Omega^{+}, r=r_{0} r_{2}$ and $R=r_{1} r_{2}$; we note that by the nature of the bipartition of the vertices of $\mathcal{M}$, the elements of $\Sigma$ are in one-to-one correspondence with the endpoints of the edges (i.e., the arcs) of the flag graph of $\mathcal{M}$. (If we instead define $\hat{r}=r_{2} r_{0}$ and $\hat{R}=r_{2} r_{1}$, then we get the "other" orientation of $\mathcal{M}$.) A fragment of an oriented map $\mathcal{M}_{0}$ is shown in Figure 1, with a portion of the underlying map $\mathcal{M}$ also shown.

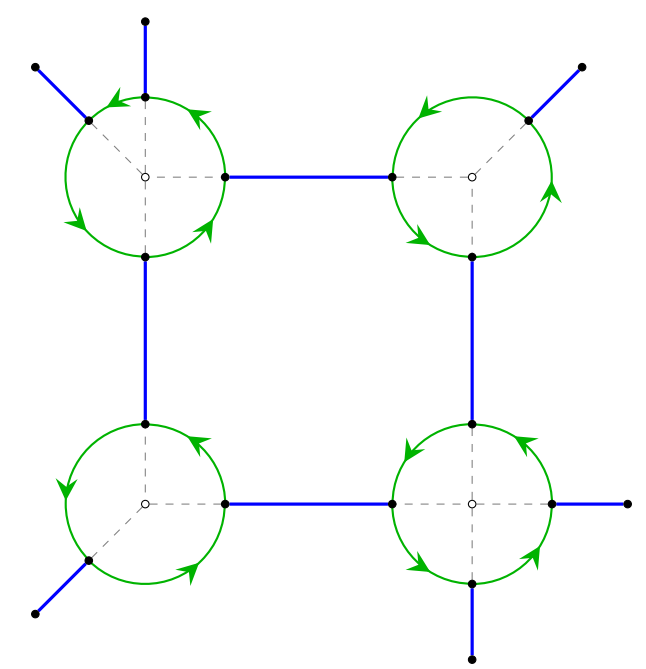

Figure 1. A fragment of an oriented map. The arcs are shown as black vertices; the green arrows correspond to the action of $R$ on the arcs, and blue edges, to the action of $r$. The dashed lines and white vertices are from the underlying unoriented map. Orientation is counterclockwise, indicated with arrows. 
Conversely, any oriented map $\mathcal{M}_{0}:=\{\Sigma,\langle r, R\rangle\}$ gives rise to the associated orientable map $\mathcal{M}=\left\{\Omega,\left\langle r_{0}, r_{1}, r_{2}\right\rangle\right\}$ in the following way: let $\Sigma_{0}$ and $\Sigma_{1}$ be two copies (colored using colors 0 and 1) of $\Sigma$, with elements $\sigma_{0}$ and $\sigma_{1}$ respectively, and define

$$
\Omega:=\Sigma_{0} \cup \Sigma_{1}
$$

$$
\begin{aligned}
& r_{0}\left(\sigma_{0}\right):=r(\sigma)_{0} \\
& r_{2}\left(\sigma_{0}\right):=r(\sigma)_{1} \\
& r_{1}\left(\sigma_{0}\right):=R(\sigma)_{1}
\end{aligned}
$$

$$
\begin{aligned}
& r_{0}\left(\sigma_{1}\right):=r(\sigma)_{1} \\
& r_{2}\left(\sigma_{1}\right):=r(\sigma)_{0} \\
& r_{1}\left(\sigma_{1}\right):=R^{-1}(\sigma)_{0}
\end{aligned}
$$

It is straightforward to show that the skeleton of an oriented map is isomorphic as a graph to the skeleton of the underlying orientable map.

Example 1. The cube may be considered either as

- an oriented map: $\mathcal{M}_{0}:=\{\Sigma,\langle r, R\rangle\}$, or

- as an (orientable) map: $\mathcal{M}=\left\{\Omega,\left\langle r_{0}, r_{1}, r_{2}\right\rangle\right\}$.

These two representations are depicted in Figure 2.
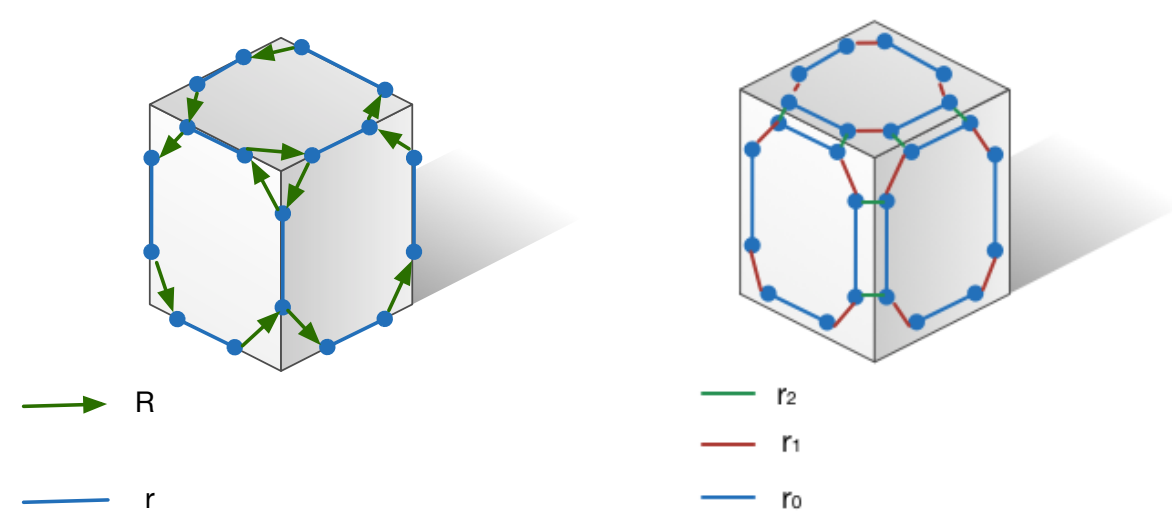

Figure 2. The cube may be considered as an oriented map determined by $R$ and $r$, or as a map determined by $r_{0}, r_{1}, r_{2}$.

We recall that two maps $\mathcal{M}^{(1)}=\left\{\Omega^{(1)},\left\langle r_{0}^{(1)}, r_{1}^{(1)}, r_{2}^{(1)}\right\rangle\right\}$ and $\mathcal{M}^{(2)}=\left\{\Omega^{(2)},\left\langle r_{0}^{(2)}, r_{1}^{(2)}, r_{2}^{(2)}\right\rangle\right\}$ are isomorphic if there exists a bijection $h$ between $\Omega^{(1)}$ and $\Omega^{(2)}$ such that $h r_{i}^{(1)}=r_{i}^{(2)} h$ for $i=0,1,2$, and we call such a bijection a map isomorphism.

Similarly, we say that two oriented maps $\mathcal{M}_{0}^{(1)}=\left\{\Sigma^{(1)},\left\langle r^{(1)}, R^{(1)}\right\rangle\right\}$ and $\mathcal{M}_{0}^{(2)}=$ $\left\{\Sigma^{(2)},\left\langle r^{(2)}, R^{(2)}\right\rangle\right\}$ are isomorphic if there exists a bijection $h$ between $\Sigma^{(1)}$ and $\Sigma^{(2)}$ such that $h r^{(1)}=r^{(2)} h$ and $h R^{(1)}=R^{(2)} h$. Such a bijection is called an oriented map isomorphism.

As usual, the isomorphisms of a structure to itself are called automorphisms and form a group. By Aut $\mathcal{M}$ we denote the group of map automorphisms, and similarly by Aut $\mathcal{M}_{o}$ we denote the group of oriented map isomorphisms.

Lemma 1 (Fundamental Lemma of Symmetries of Maps). The action of the automorphism group Aut $\mathcal{M}$ of the map $\mathcal{M}=\left\{\Omega,\left\langle r_{0}, r_{1}, r_{2}\right\rangle\right\}$ is semi-regular on the set of flags $\Omega$.

Lemma 2 (Fundamental Lemma of Symmetries of Oriented Maps). The action of the automorphism group Aut $\mathcal{M}_{0}$ of the oriented map $\mathcal{M}=\{\Sigma,\langle r, R\rangle\}$ is semi-regular on the set of arcs $\Sigma$. 
The validity of both lemmas follows from the fact that for connected structures, any element (flag or arc) can be mapped to any other element in at most one way. Namely, if the image $w$ of a given element $v$ is chosen, then the images of all its neighbours are uniquely determined. By repeating the argument, either the full automorphism is constructed or a contradiction proves that no automorphism mapping $v$ to $w$ exists.

Definition 1. If the automorphism group of a map has $k$ orbits, the map is called a k-orbit map.

Definition 2. If the automorphism group of an oriented map has $k$ orbits, the oriented map is called a k-orbit oriented map.

The Fundamental Lemmas of Symmetries of Maps and Oriented Maps have several interesting consequences that follow by the application of basic permutation group theory:

Corollary 1. Let $\mathcal{M}=\left\{\Omega,\left\langle r_{0}, r_{1}, r_{2}\right\rangle\right\}$ be a map.

- $\quad$ The cardinality of each orbit of Aut $\mathcal{M}$ on $\Omega$ is equal to the order of Aut $\mathcal{M}$.

- $\mid$ Aut $\mathcal{M} \mid$ is a divisor of $|\Omega|$.

- $\quad$ The projection $\mathcal{M} \rightarrow \mathcal{M} /$ Aut $\mathcal{M}$ is a regular covering projection.

- $\quad$ The quotient $T(\mathcal{M})=\mathcal{M} /$ Aut $\mathcal{M}=\left\{\Omega^{\prime},\left\langle r_{0}^{\prime}, r_{1}^{\prime}, r_{2}^{\prime}\right\rangle\right\}$ is a degenerate flag graph, called the symmetry type graph.

- $\quad$ The order $k=\left|\Omega^{\prime}\right|$ of $T(\mathcal{M})$ is equal to $k=|\Omega| / \mid$ Aut $\mathcal{M} \mid$, and $\mathcal{M}$ is a k-orbit map.

For the definition of a regular covering projection, the reader is referred, for example, to [1].

Symmetry type graphs have been used previously in the analysis of maps; see, for instance, [5-9] . We extend these results of maps to oriented maps in the obvious way by introducing a useful tool that we call an oriented symmetry type graph (see Figure 3):

Corollary 2. Let $\mathcal{M}_{o}=\{\Sigma,\langle R, r\rangle\}$ be an oriented map.

- $\quad$ The cardinality of each orbit of $A u t \mathcal{M}_{0}$ on $\Sigma$ is equal to the order of $A u t \mathcal{M}_{0}$.

- $\mid$ Aut $\mathcal{M}_{o} \mid$ is a divisor of $|\Sigma|$.

- The projection $\mathcal{M}_{0} \rightarrow \mathcal{M}_{0} /$ Aut $\mathcal{M}_{0}$ is a regular covering projection.

- The quotient $T_{0}\left(\mathcal{M}_{0}\right)=\mathcal{M}_{0} /$ Aut $\mathcal{M}_{0}=\left\{\Sigma^{\prime},\left\langle R^{\prime}, r^{\prime}\right\rangle\right\}$ is a degenerate arc graph, called the oriented symmetry type graph.

- $\quad$ The order $k_{o}=\left|\Sigma^{\prime}\right|$ of $T_{o}\left(\mathcal{M}_{o}\right)$ is equal to $k_{o}=|\Sigma| / \mid$ Aut $\mathcal{M}_{o} \mid$, and $\mathcal{M}_{o}$ is a $k_{0}$-orbit oriented map.

We now demonstrate an application of symmetry type graphs and oriented symmetry type graphs. A map is regular (in the strong sense, i.e., the order of its automorphism group is $\mid$ Aut $\mathcal{M}|=| \Omega|=4| E \mid$ ) if and only if its symmetry type graph is a one-vertex graph $(k=1)$. Similarly, an oriented map is regular (i.e., the order if its orientation-preserving automorphism group is $\mid$ Aut $\mathcal{M}_{o}|=| \Sigma|=2| E \mid$ ) if and only if its symmetry type graph is a one-vertex graph $\left(k_{o}=1\right)$. We note that a regular oriented map may be either a regular or chiral (orientable) map.

In general, the underlying orientable map of a $k_{0}$-orbit oriented map is either a $k_{0}$-or $2 k_{0}$-orbit map. 

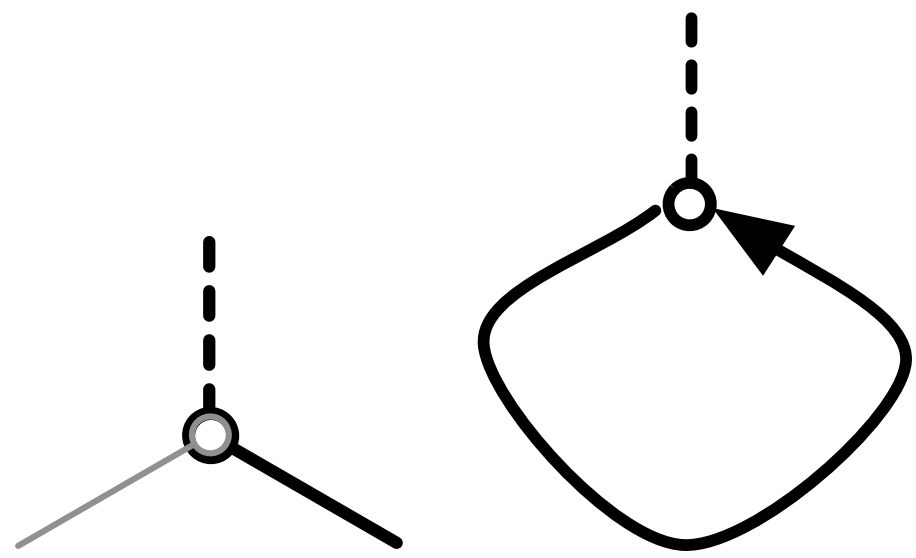

Figure 3. An example of a symmetry type graph of a regular map and an oriented symmetry type graph of an oriented regular map; in this case, they may be viewed as the symmetry/oriented symmetry type graph of the cube.

Example 2. The $n$-sided pyramids for $n>3$ all have the the same symmetry type graph and the same oriented symmetry type graph. The case $n=4$ is depicted in Figure 4 .

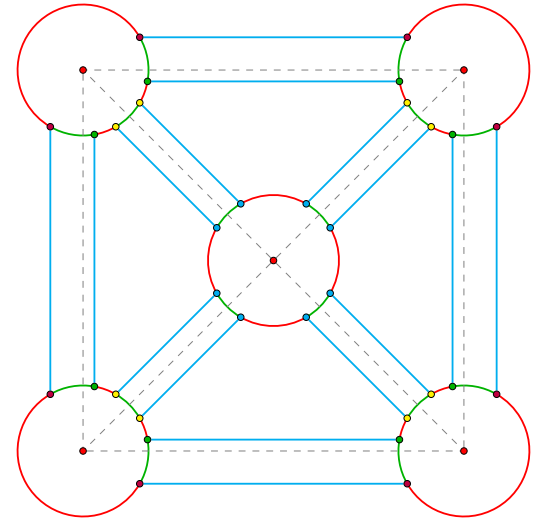

(a) The flag graph of the square pyramid $\mathcal{M}$.

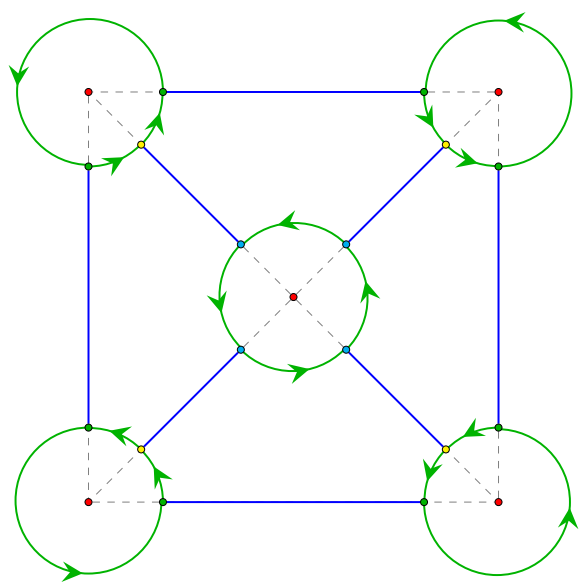

(c) The arc graph of the square pyramid $\mathcal{M}_{0}$.

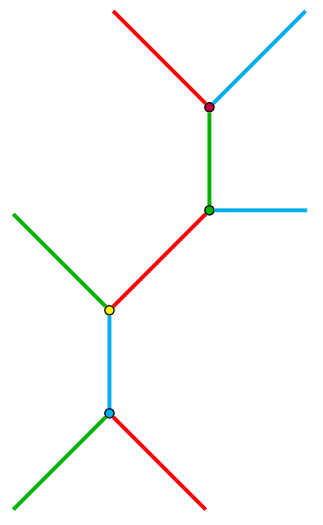

(b) The quotient $T(\mathcal{M})$.

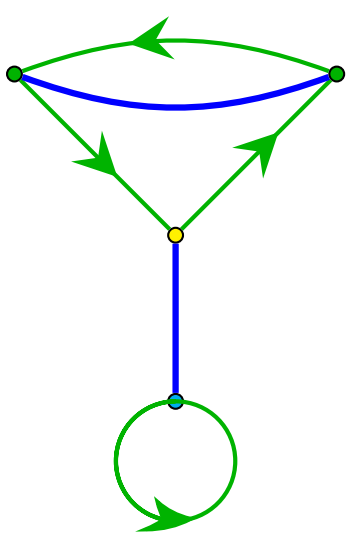

(d) The quotient $T\left(\mathcal{M}_{0}\right)$.

Figure 4. The four-sided pyramid represented as a map $\mathcal{M}$ and as an oriented map $\mathcal{M}_{0}$, with the corresponding symmetry type graph $T(\mathcal{M})$ and oriented symmetry type graph $T_{o}\left(\mathcal{M}_{o}\right)$. 


\section{Operations On Oriented Maps}

Operations on maps with the property that the new map resides in the same surface as the original map have been the subject of active investigation (see [6,7,10-13]). If the underlying surface is orientable, we may choose one orientation that induces an orientation of the corresponding map, making it an oriented map. If we keep the surface orientation, the operation may be shifted from maps to oriented maps. In the context of this paper, we work entirely with the generating permutations $r$ and $R$ of the oriented map to describe these operations.

Each operation $0 \mathrm{p}$ in this paper can be described in the following way. Each $\operatorname{arc} \sigma$ of the original oriented map $\mathcal{M}_{0}=\{\Sigma,\langle r, R\rangle\}$ gives rise to $k$ arcs $\sigma_{0}, \sigma_{1}, \ldots, \sigma_{k-1}$ of the resulting oriented map $\mathrm{Op}\left(\mathcal{M}_{0}\right):=\left\{\Sigma^{\prime},\left\langle r^{\prime}, R^{\prime}\right\rangle\right\}$, where $\Sigma^{\prime}=\Sigma_{0} \cup \Sigma_{1} \cup \ldots \Sigma_{k-1}$ and each $\Sigma_{i}$ consists of copies of arcs of $\Sigma$ with subscript $i$. If $r$ is the original involution and $R$ is the original permutation of $\mathcal{M}_{0}$, we denote by $r^{\prime}$ and $R^{\prime}$ the respective permutations of the resulting map $0 \mathrm{p}\left(\mathcal{M}_{o}\right)$, which reside in the same surface as $\mathcal{M}_{0}$ with the same orientation as $\mathcal{M}_{0}$. Because $\left|\Sigma^{\prime}\right|=k|\Sigma|$ we call $k$ the edge-multiplier of Op. We derive the action of five operations, Dual (Du), Medial (Me), Truncation ( $\mathrm{Tr}), \mathrm{Snub}(\mathrm{Sn})$, and Chamfer (Ch), by considering directly their local effect on the vertices and edges of an oriented map $\mathcal{M}_{0}$. Each of these operations is well known from the study of polyhedra. We present the definition of $r^{\prime}$ and $R^{\prime}$ for each of these operations, by describing their action on the $k \operatorname{arcs} \sigma_{1}, \ldots, \sigma_{k}$ of $\operatorname{Op}\left(\mathcal{M}_{0}\right)$ associated with each $\operatorname{arc} \sigma$ of $\mathcal{M}_{0}$. Illustrations corresponding to the derivation of each of these new $r^{\prime}$ and $R^{\prime}$ are given in the corresponding figures.

\subsection{Orientation Reversal Re or *}

An operation with edge-multiplier 1 that can be defined on oriented maps but has no counterpart on maps is the orientation reversal, Re. Given an oriented map $\mathcal{M}_{0}=\{\Sigma,\langle r, R\rangle\}$, the reversal is given by $\mathcal{M}_{o}^{*}:=\left\{\Sigma,\left\langle r, R^{-1}\right\rangle\right\}$. In other words, we have the following:

$$
r^{\prime}\left(\sigma_{0}\right)=r(\sigma)_{0} \quad R^{\prime}\left(\sigma_{0}\right)=R^{-1}(\sigma)_{0} \quad \operatorname{Re}
$$

\subsection{Dual Du and Improper Dual IDu}

The definition of dual seems that it should be straightforward. We are tempted to define it as follows:

$$
r^{\prime}\left(\sigma_{0}\right)=r(\sigma)_{0} \quad R^{\prime}\left(\sigma_{0}\right)=R(r(\sigma))_{0} \quad \text { IDu }
$$

However, if we draw the corresponding figure, it becomes obvious that the map defined by the above rules is oriented in the opposite direction from the original map. Hence, we call it the improper dual IDu. A local portion of a map, along with the derivation of its $\mathrm{Du}$ and IDu, is shown in Figure 5.

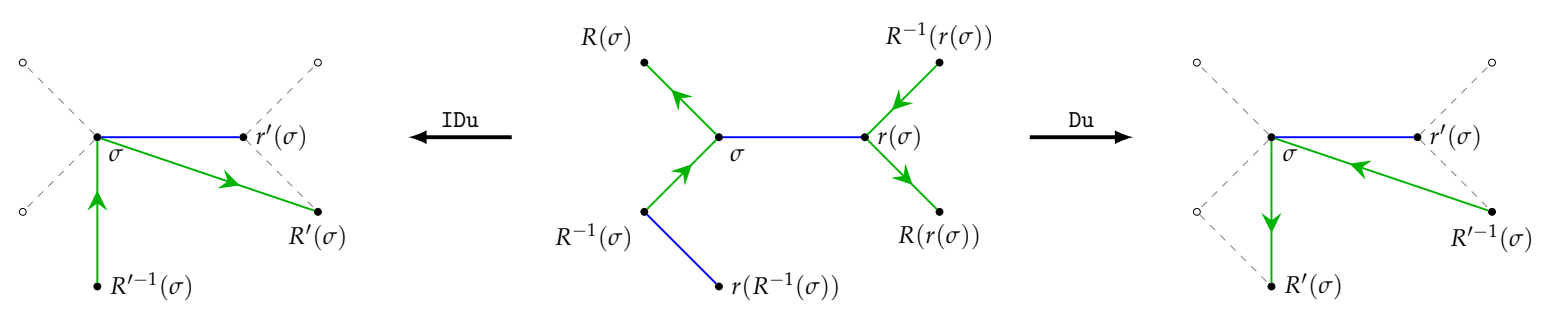

Figure 5. Improper dual (IDu) and dual (Du) of an oriented map, local figure. In the center is a local portion of an oriented map, to the left is is the local picture of IDu, and to the right is Du. Because there is only one copy of $\Sigma$ used in the construction of IDu and Du, we have suppressed the 0 subscript on the arcs for clarity. 
The correct definition of the orientation preserving dual Du is as follows:

$$
r^{\prime}\left(\sigma_{0}\right)=R\left(r\left(R^{-1}(\sigma)\right)\right)_{0} \quad \quad R^{\prime}\left(\sigma_{0}\right)=r\left(R^{-1}(\sigma)\right)_{0} \quad \mathrm{Du}
$$

\subsection{Truncation $(\mathrm{Tr})$}

The truncation operation is a well-known operation that has been applied to polyhedra and maps on many occasions; see, for instance, [14]. It is derived from the geometric operation in which the vertices of a convex polyhedron are cut off shallowly to form a new polyhedron. Here, we present it in the oriented version. Each arc $\sigma$ of the oriented map $\mathcal{M}_{0}:=\{\Sigma,\langle r, R\rangle\}$ gives rise to three $\operatorname{arcs} \sigma_{0}, \sigma_{1}$ and $\sigma_{2}$ of the truncated oriented map $\operatorname{Tr}\left(\mathcal{M}_{0}\right):=\left\{\Sigma^{\prime},\left\langle r^{\prime}, R^{\prime}\right\rangle\right\}$. Hence the edge-multiplier of $\operatorname{Tr}$ is 3 . See Figure 6. We define $r^{\prime}$ and $R^{\prime}$ as follows:

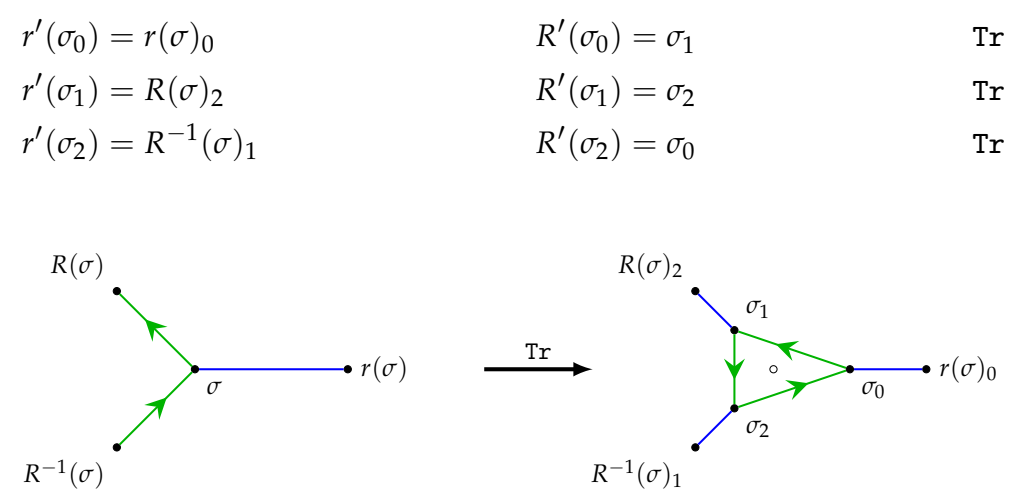

Figure 6. Truncated oriented map $\operatorname{Tr}$, local figure.

\subsection{Medial (Me)}

Another operation of the same type is the medial Me, with edge-multiplier 2. It is derived from the geometric operation in which the vertices of a convex polyhedron are cut off at the midpoints of the edges (sometimes called full truncation or rectification) to form a new polyhedron. For example, the medial of a cube is the cuboctahedron. The medial of ordinary maps has been studied in [5]. The medial of an oriented map is defined as follows (see Figure 7):

$$
\begin{array}{lll}
r^{\prime}\left(\sigma_{0}\right)=R(\sigma)_{1} & R^{\prime}\left(\sigma_{0}\right)=\sigma_{1} & \mathrm{Me} \\
r^{\prime}\left(\sigma_{1}\right)=R^{-1}(\sigma)_{0} & R^{\prime}\left(\sigma_{1}\right)=r(\sigma)_{0} & \text { Me }
\end{array}
$$
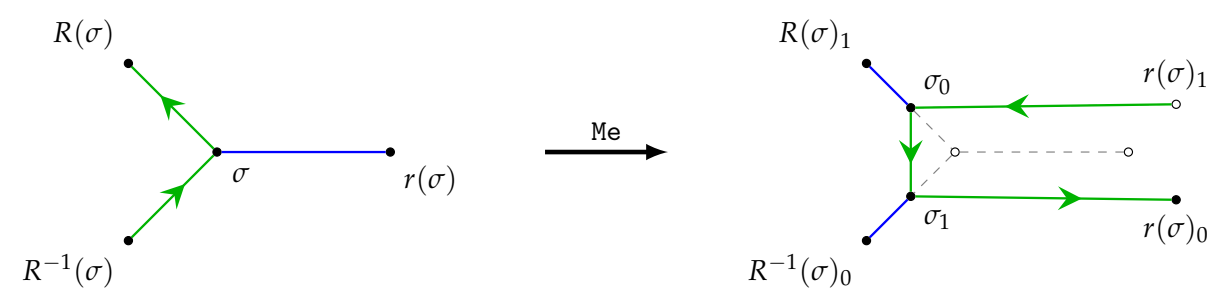

Figure 7. Medial Me of an oriented map, local figure.

\subsection{Snub (Sn)}

The snub is an operation with edge-multiplier 5 that can only be defined on oriented (or orientable) maps; see Figure 8. Geometrically, it corresponds to the new polyhedron formed by moving all faces of a convex polyhedron outwards, twisting each face about its center, and adding pairs of triangular 
faces in place of the original edges. We note that definitions provided by Conway (discussed in [10]) and Coxeter [14] differ slightly.

$$
\begin{array}{lll}
r^{\prime}\left(\sigma_{0}\right)=r(\sigma)_{4} & R^{\prime}\left(\sigma_{0}\right)=\sigma_{1} & \mathrm{Sn} \\
r^{\prime}\left(\sigma_{1}\right)=r(\sigma)_{1} & R^{\prime}\left(\sigma_{1}\right)=\sigma_{2} & \mathrm{Sn} \\
r^{\prime}\left(\sigma_{2}\right)=\sigma_{3} & R^{\prime}\left(\sigma_{2}\right)=R^{-1}(\sigma)_{3} & \mathrm{Sn} \\
r^{\prime}\left(\sigma_{3}\right)=\sigma_{2} & R^{\prime}\left(\sigma_{3}\right)=\sigma_{4} & \mathrm{Sn} \\
r^{\prime}\left(\sigma_{4}\right)=r(\sigma)_{0} & R^{\prime}\left(\sigma_{4}\right)=R(\sigma)_{0} & \mathrm{Sn}
\end{array}
$$

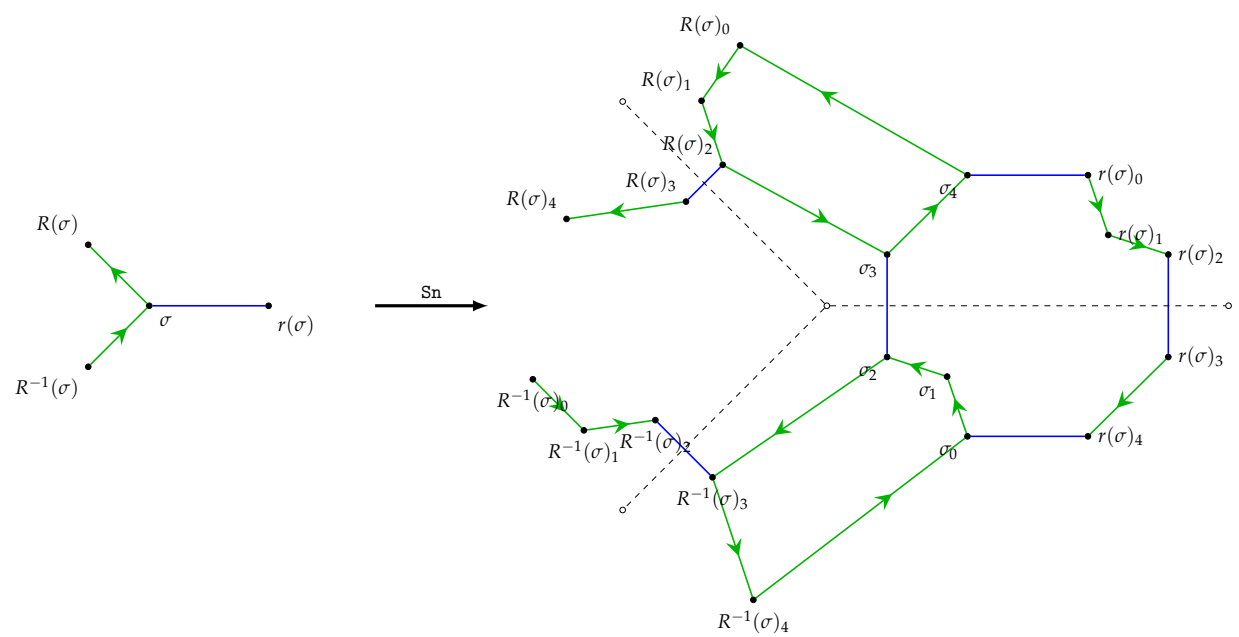

Figure 8. Snub Sn of an oriented map, local figure.

\subsection{Chamfer $\mathrm{Ch}$}

The chamfer operation is an operation with edge-multiplier 4; see Figure 9. Geometrically, it is derived from shallowly slicing off the edges of a convex polyhedron. As a map operation, it was used, for instance, in [8].

$$
\begin{aligned}
& r^{\prime}\left(\sigma_{0}\right)=\sigma_{1} \\
& r^{\prime}\left(\sigma_{1}\right)=\sigma_{0} \\
& r^{\prime}\left(\sigma_{2}\right)=\sigma_{3} \\
& r^{\prime}\left(\sigma_{3}\right)=\sigma_{2}
\end{aligned}
$$$$
R^{\prime}\left(\sigma_{0}\right)=R(\sigma)_{0}
$$$$
\mathrm{Ch}
$$$$
R^{\prime}\left(\sigma_{1}\right)=\sigma_{2}
$$$$
R^{\prime}\left(\sigma_{2}\right)=r(R(\sigma))_{3}
$$$$
R^{\prime}\left(\sigma_{3}\right)=R^{-1}(r(\sigma))_{1}
$$
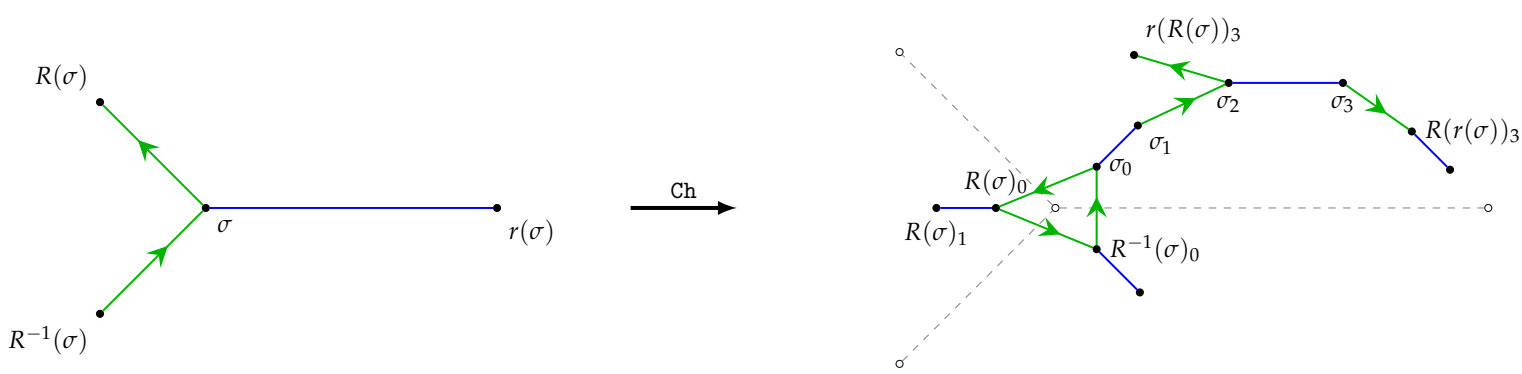

Figure 9. Chamfer $\mathrm{Ch}$ of an oriented map, local figure.

\subsection{One-Dimensional Subdivision Su1}

The one-dimensional subdivision is an operation with edge-multiplier 2. It is a simple operation that subdivides each edge of the oriented map. For maps, it has been used in several places in 
the literature (e.g., [11-13]). It is a building block for composite operations. The following is a formal definition:

$$
\begin{array}{lll}
r^{\prime}\left(\sigma_{0}\right)=\sigma_{1} & R^{\prime}\left(\sigma_{0}\right)=R(\sigma)_{0} & \text { Su1 } \\
r^{\prime}\left(\sigma_{1}\right)=\sigma_{0} & R^{\prime}\left(\sigma_{1}\right)=r(\sigma)_{1} & \text { Su1 }
\end{array}
$$

\subsection{Composite Operations}

Given two operations $0 \mathrm{p} 1$ and $0 \mathrm{p} 2$, it is natural to consider the operation $0 \mathrm{p}$ that is obtained by first applying $0 \mathrm{p} 1$ and then $\mathrm{Op} 2$; that is, $\mathrm{Op}=0 \mathrm{p} 2 \mathrm{Op} 1$. It is not hard to see that the edge-multiplier of $0 \mathrm{p}$ is the product of edge-multipliers of $0 \mathrm{p} 1$ and $0 \mathrm{p} 2$. The following are some examples:

$$
\begin{aligned}
\mathrm{Su} 2 & =\mathrm{Du} \operatorname{Tr} \mathrm{Du} \\
\mathrm{BS} & =\mathrm{Su} 2 \mathrm{Su} 1 \\
\mathrm{Le} & =\mathrm{Tr} \mathrm{Du} \\
\mathrm{Co} & =\mathrm{Du} \mathrm{BS} \\
\mathrm{An} & =\mathrm{Du} \mathrm{Me} \\
\mathrm{Go} & =\mathrm{Du} \mathrm{Me} \mathrm{Tr}
\end{aligned}
$$

Each of these operations has been used for maps. One can use the same formulae to define them for oriented maps. Two-dimensional subdivision is sometimes called omni-capping. Each face of the original map is subdivided into triangles by placing a new vertex in the center of the face and joining it to each of the original vertices. Leapfrogging is an operation that has been studied extensively in theoretical chemistry; see, for instance, [11]. For instance, the leapfrog of a dodecahedron is a truncated icosahedron. In general, a leapfrog of a fullerene is another fullerene (see, e.g., [11]). In a similar way, An has been used in [15] and Go has been used in [7]. The edge-multiplier for Su2 is 3; for BS, Co and Go, the edge-multiplier is 6; and the edge-multiplier for An is 2. An early use of An can be found in [16], where it is called the web graph. In [17], it is called the radial graph, and its dual Me is also discussed. In [11-13], several operations on maps are discussed.

\section{Some Properties of the Operations and $k$-Orbit Oriented Maps}

There is an important paper by Orbanić, Pellicer and Weiss [18] in which, among other things, the following theorem is proved.

Theorem 1. If $\mathcal{M}$ is a k-orbit map, then its truncation $\operatorname{Tr}(\mathcal{M})$ is either a $3 k$-, 3k/2-or k-orbit map.

Motivated by [18], we define the following:

Definition 3. Let Op be a map operation. If there exists a k-orbit map $\mathcal{M}$ such that $\mathrm{Op}(\mathcal{M})$ is a $\lambda k$-orbit map, then $\lambda$ is called a flag-orbit multiplier. By $\Lambda(\mathrm{Op})$ we denote the set of flag-orbit multipliers of $0 \mathrm{p}$.

For instance, $\Lambda(\operatorname{Tr})=\{1,3 / 2,3\}$.

Problem 1. Investigate sets of flag-orbit multipliers for various operations on maps.

So far, flag-orbit multipliers have been investigated for truncation, medials and chamfering $[5,8,9,18]$. One could consider these operations on oriented maps, that is, as follows:

Definition 4. Let $0 \mathrm{p}$ be an oriented-map operation. If there exists a $k$-orbit oriented map $\mathcal{M}_{o}$ such that $\mathrm{Op}\left(\mathcal{M}_{0}\right)$ is a $\lambda_{o} k$-orbit oriented map, then $\lambda_{o}$ is called an arc-orbit multiplier. By $\Lambda_{o}(\mathrm{Op})$ we denote the set of arc-orbit multipliers of $0 \mathrm{p}$. 
Problem 2. Investigate sets of arc-orbit multipliers for various operations on oriented maps.

In the case of maps, the main tools are symmetry type graphs. In the case of oriented maps, it seems natural that we should use oriented symmetry type graphs.

We note that the operations $\mathrm{Op}$, such as $\mathrm{Tr}, \mathrm{Me}, \mathrm{Du}$, and $\mathrm{Ch}$, have been studied for general maps, and in the context of oriented maps, they satisfy the following condition: $\operatorname{Op}\left(\mathcal{M}_{0}\right) \cong \mathrm{Op}\left(\mathcal{M}_{0}^{*}\right)^{*}$. We call such operations amphicheiral — equivalent to their mirror image. On the other hand, this is not the case for the snub operation. We may define a different snub operation $\operatorname{Sn}^{*}\left(\mathcal{M}_{0}\right):=\operatorname{Sn}\left(\mathcal{M}_{0}^{*}\right)^{*}$. Operations that are not amphicheiral are called chiral. Therefore, the snub is a chiral operation. The snub can only be defined for oriented maps. It certainly cannot be defined on a non-orientable map. If we try to define it for an orientable map, there is no way to distinguish between $S n^{*}$ and $S n$; hence we have to define them both simultaneously and name them arbitrarily.

We may carry this idea to oriented maps and to their oriented symmetry type graphs. We say that an oriented map or oriented symmetry type graph is amphicheiral if $\mathcal{M}_{o} \cong \mathcal{M}_{o}^{*}$, or $T\left(\mathcal{M}_{o}\right) \cong T\left(\mathcal{M}_{0}^{*}\right)$. Otherwise, it is chiral (in the sense of Conway). In practice, we may check whether a map is chiral by reversing the directions of arrows and checking whether the resulting map is orientably isomorphic to the original or not. All oriented symmetry type graphs up to four vertices are amphicheiral. The smallest chiral graph is depicted in Figure 10.
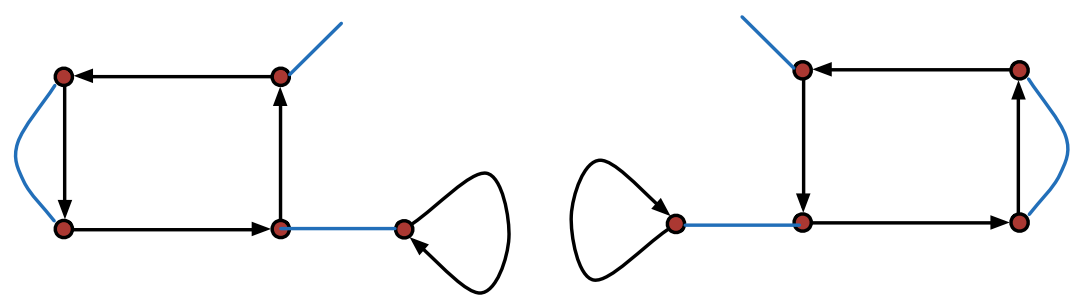

Figure 10. Smallest chiral oriented symmetry type graph (both versions).

\section{Edge-Transitive Oriented Maps}

Recently it has been shown that symmetry type graphs are quite a powerful tool when studying certain types of maps. For instance, they provide combinatorial, group-free approaches to the question of classifying edge-transitive maps. In [19], Graver and Watkins classified edge-transitive maps into 14 distinct types. In [20], Širán̆, Tucker and Watkins showed that each of the 14 types admits a realization by an oriented map. In [6], Orbanić et al. showed that the 14 types can naturally be described by 14 symmetry type graphs, shown in Figure 11.

The main idea of the proof is to use the fact that in the edge-transitive case, the spanning subgraph of the symmetry type graph determined by $r_{0}, r_{2}$ must be connected. There are five possibilities, depicted in Figure 12. By inserting the edges of $r_{1}$ in all possible ways, one obtains exactly the 14 types.

Karabáš [21] gives a list of low-genus orientable edge-transitive maps. Eight types of these maps are mentioned. Karabáš and Pisanski [22] have shown that these eight classes correspond to eight different oriented symmetry type graphs.

We note that for an oriented map, the number of edge orbits according to the orientation-preserving automorphism group corresponds to the number of edges arising from $r^{\prime}$ in the respective oriented symmetry type graph. This limits the search to oriented symmetry type graphs on one or two vertices. We call such oriented maps edge-transitive in the strong sense. 


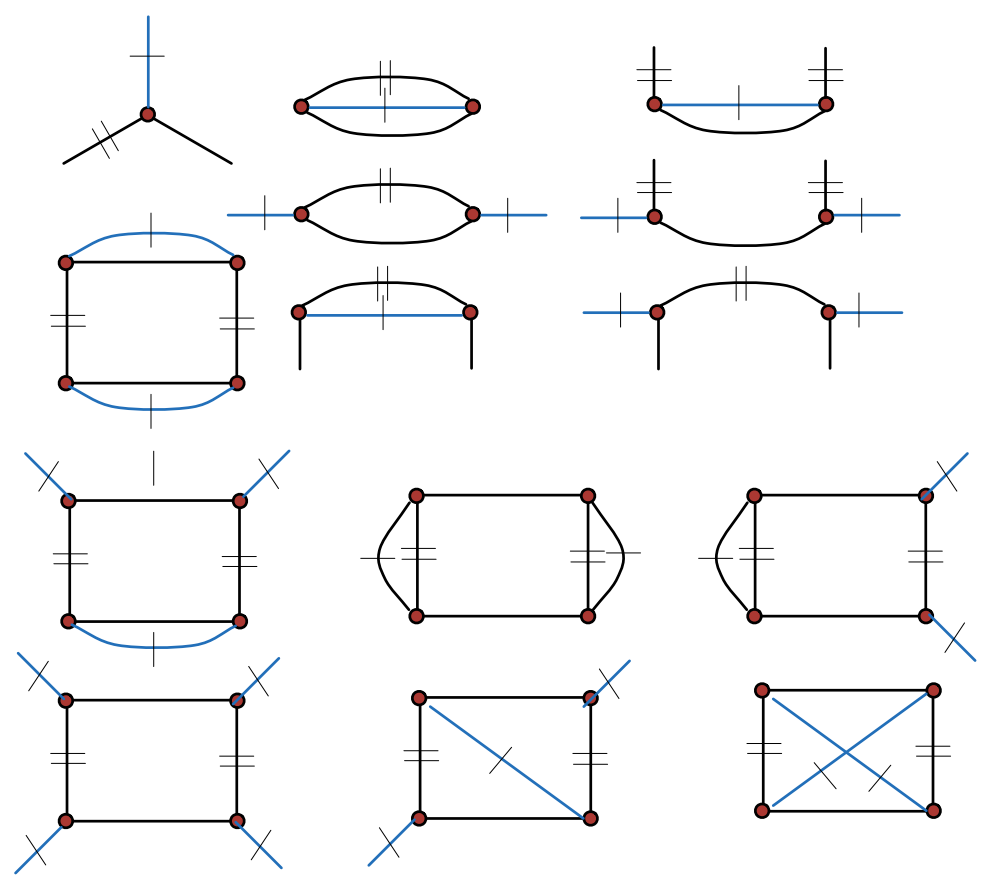

Figure 11. The 14 symmetry type graphs of edge-transitive maps.

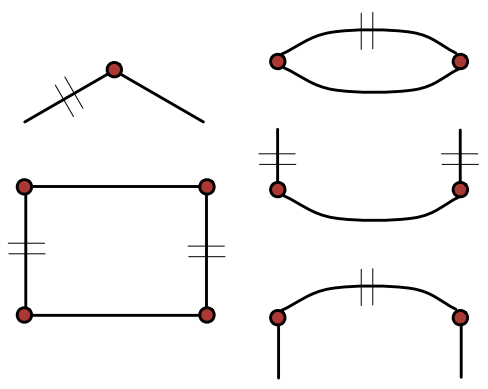

Figure 12. Five possible quotients of an edge quadrangle.

On the other hand, an oriented map may not be edge-transitive in the strong sense, but it may have an edge-transitive underlying (orientable) map. Such a map is edge-transitive in the weak sense. One can classify such maps.

Theorem 2. ([22]) An oriented map is edge-transitive in the weak sense if and only if its oriented symmetry type graph has two edges corresponding to $r$ and there exists an extended automorphism reversing the arrows of $R$ that interchanges the two $r$-edges.

This, in turn, implies that a weak edge-transitive oriented map has at most four arc symmetry classes, or equivalently, that its oriented symmetry type graph has at most four vertices. Figure 13 depicts all 17 oriented symmetry type graphs on at most four vertices. In addition to three strong edge-transitive types, we obtain five weak edge-transitive types.

This rather simple combinatorial approach complements the much more sophisticated group-theoretic approach by Jones [23]. 


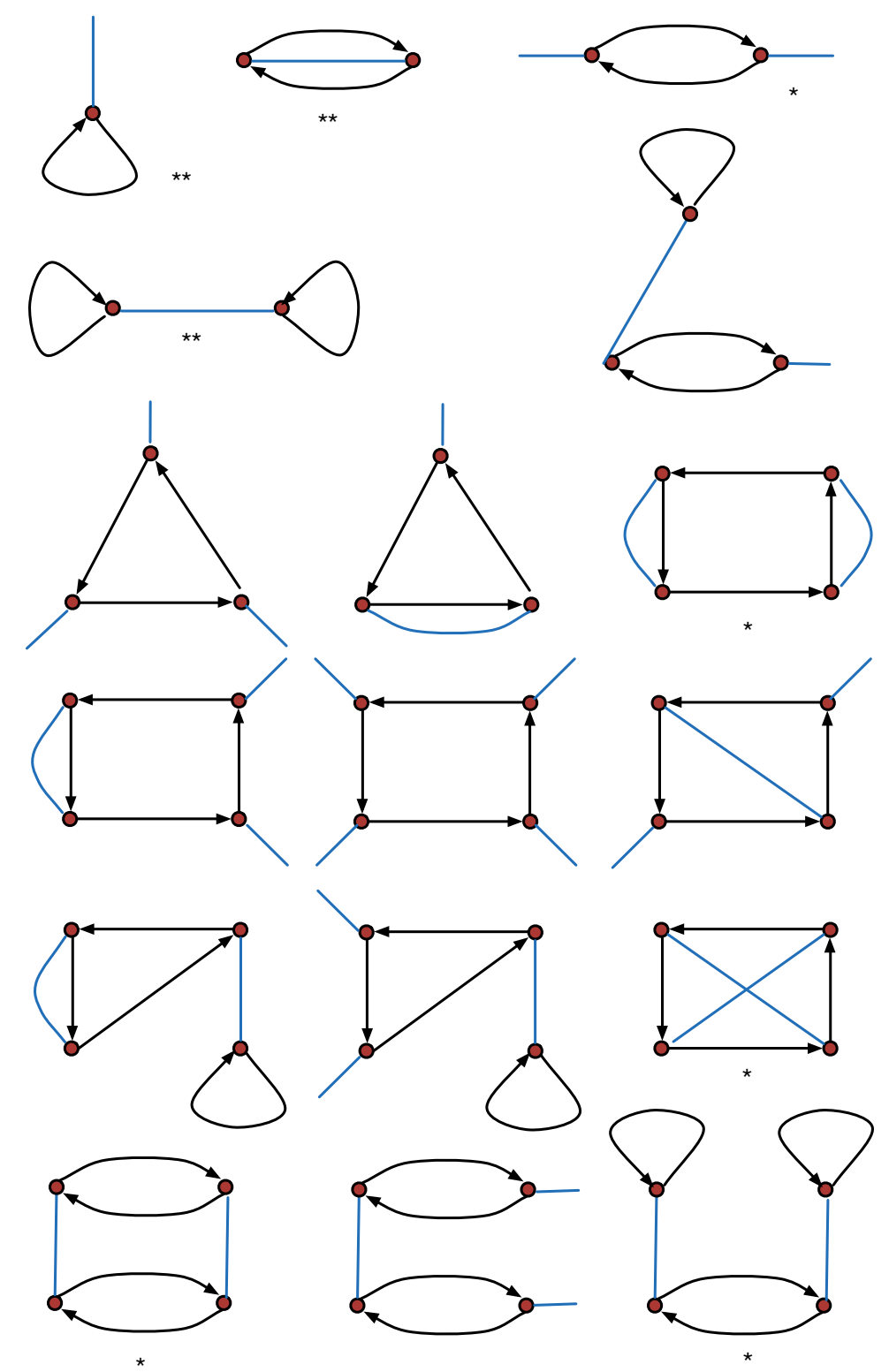

Figure 13. The 17 oriented symmetry type graphs on at most four vertices. Eight of these marked by $\left(^{* *}\right)$ or $\left(^{*}\right)$ correspond to edge-transitive maps. The three marked by $\left({ }^{* *}\right)$ correspond to the strong edge-transitive maps.

\section{Conclusions}

In this paper, we described the basics of operations on oriented maps and their oriented symmetry type graphs. It would be interesting to carry over the investigations that were performed for similar operations on maps and their symmetry types, such as those by Orbanić et al. [6], del Rio Francos [8,9], and Hubard et al. [5] to oriented maps and their oriented symmetry type graphs. Another context in which it would be interesting to investigate similar questions would be in the higher ranks provided by maniplexes and oriented maniplexes (cf., Cunningham et al. [24]).

Finally, there exists a collection of operations on polyhedra, described using Conway polyhedron notation $[10,25]$, originally developed by John $\mathrm{H}$. Conway but later promoted and expanded by George $\mathrm{W}$. Hart, that can be readily carried over to maps and oriented maps. We believe that our approach via flag graphs and arc graphs should be applied to Conway operations to give them rigorous definitions. Namely, to the best of our knowledge, currently only verbal and pictorial descriptions of Conway 
operations are available. A dictionary that would translate between the two notations would certainly be useful. The reader is referred to [26-30] for related work and for further references. In particular, this theory can be extended to hypermaps [28,31].

Acknowledgments: T. Pisanski's research was supported in part by the ARRS of Slovenia under grants: P1-0294 and N1-0032. Leah W. Berman's research was supported by the Simons Foundation (Grant \# 209161 to L. Berman). Gordon Williams and Leah Berman would like to thank the Faculty of Mathematics and Physics at the University of Ljubljana for its hospitality and support during our sabbatical stay in Slovenia.

Author Contributions: Co-authors are listed alphabetically as usual in mathematics. Each author contributed substantially to the work.

Conflicts of Interest: The authors declare no conflict of interest.

\section{References}

1. Gross, J.L.; Tucker, T.W. Topological Graph theory; Dover Publications Inc.: Mineola, NY, USA, 2001.

2. Pisanski, T.; Potočnik, P. Handbook of Graph Theory; Chapter Graphs on Surfaces; CRC Press: Boca Raton, FL, USA, 2003.

3. Godsil, C.; Royle, G. Algebraic graph theory. In Graduate Texts in Mathematics; Springer: New York, NY, USA, 2001; Volume 207.

4. Nedela, R. Maps, Hypermaps and Related Topics, July 2007. Available online: http://www.savbb.sk/ $\sim$ nedela/CMbook.pdf (accessed on 3 July 2017).

5. Hubard, I.; del Río Francos, M.; Orbanić, A.; Pisanski, T. Medial symmetry type graphs. Electron. J. Comb. 2013, 20, 28.

6. Orbanić, A.; Pellicer, D.; Pisanski, T.; Tucker, T.W. Edge-transitive maps of low genus. Ars Math. Contemp. 2011, 4, 385-402.

7. Pisanski, T.; Servatius, B. Configurations from a Graphical Viewpoint; Springer: New York, NY, USA, 2013.

8. Del Río Francos, M. Chamfering operation on k-orbit maps. Ars Math. Contemp. 2014, 7, 507-524.

9. Del Río Francos, M. Truncation symmetry type graphs. Ars Comb. 2017, 134, 135-167.

10. Conway, J.H.; Burgiel, H.; Goodman-Strauss, C. The Symmetries of Things; A K Peters Ltd.: Wellesley, MA, USA, 2008.

11. Fowler, P.; Pisanski, T. Leapfrog transformations and polyhedra of clar type. J. Chem. Soc. Faraday Trans. 1994, 90, 2865-2871.

12. Pisanski, T.; Randić, M. Bridges between geometry and graph theory. In Geometry at Work; Mathematical Association of America: Washington, DC, USA, 2000; Volume 53, pp. 174-194.

13. Pisanski, T.; Žitnik, A. Representing graphs and maps. In Topics in Topological Graph Theory; Cambridge University Press: Cambridge, UK, 2009; Volume 128, pp. 151-180.

14. Coxeter, H.S.M. Regular Polytopes; Dover Publications: Mineola, NY, USA, 1973.

15. Mohar, B.; Rosenstiel, P. Tessellation and visibility representations of maps on the torus. Discret. Comput. Geom. 1998, 19, 249-263.

16. Pisanski, T.; Malnič, A. The Diagonal Construction and Graph Embeddings; Graph Theory (Novi Sad, 1983); University of Novi Sad: Novi Sad, Serbia, 1984; pp. 271-290.

17. Negami, S.; Tucker, T.W. Bipartite polyhedral maps on closed surfaces are distinguishing 3-colorable with few exceptions. Graphs Comb. 2017, doi:10.1007/s00373-017-1788-1.

18. Orbanić, A.; Pellicer, D.; Weiss, A.I. Map operations and k-orbit maps. J. Comb. Theory Ser. A 2010, 117, $411-429$.

19. Graver, J.E.; Watkins, M.E. Locally Finite, Planar, Edge-Transitive Graphs; American Mathematical Soc.: Providence, RI, USA, 1997.

20. Širáň, J.; Tucker, T.W.; Watkins, M.E. Realizing finite edge-transitive orientable maps. J. Graph Theory 2001, $37,1-34$.

21. Karabáš, J. Edge Transitive Maps on Orientable Surfaces, 2012. Available online: http://www.savbb.sk/ $\sim$ karabas/science.html (accessed on 3 July 2017)

22. Karabáš, J.; Pisanski, T. Symmetries of Maps and Oriented Maps via Action Graphs; 2015, in preparation.

23. Jones, G.A. Automorphism groups of edge transitive maps. arXiv 2016, arXiv:1605.09461. 
24. Cunningham, G.; del Río-Francos, M.; Hubard, I.; Toledo, M. Symmetry type graphs of polytopes and maniplexes. Ann. Comb. 2015, 19, 243-268.

25. Wikipedia. Conway Polyhedron Notation. Available online: https://en.wikipedia.org/wiki/Conway_ polyhedron_notation (accessed on 3 July 2017).

26. Breda d'Azevedo, A.; Catalano, D.; Karabáš, J.; Nedela, R. Maps of Archimedean class and operations on dessins. Discrete Math. 2015, 338, 1814-1825.

27. Girondo, E. Multiply quasiplatonic Riemann surfaces. Exp. Math. 2003, 12, 463-475.

28. James, L.D. Operations on hypermaps, and outer automorphisms. Eur J. Comb. 1988, 9, 551-560.

29. Karabáš, J.; Nedela, R. Archimedean maps of higher genera. Math. Comput. 2012, 81, 569-583.

30. Singerman, D.; Syddall, R.I. The Riemann surface of a uniform dessin. Beiträge Algebra Geom. 2003, 44, 413-430.

31. Singerman, D. Riemann surfaces, Belyi functions and hypermaps. In Topics on Riemann surfaces and Fuchsian groups (Madrid, 1998); Cambridge University Press: Cambridge, UK, 2001; pp. 43-68.

(C) 2017 by the authors. Licensee MDPI, Basel, Switzerland. This article is an open access article distributed under the terms and conditions of the Creative Commons Attribution (CC BY) license (http:/ / creativecommons.org/licenses/by/4.0/). 